\title{
Drift simulation of MH370 debris using superensemble techniques
}

\author{
Eric Jansen ${ }^{1}$, Giovanni Coppini ${ }^{1}$, and Nadia Pinardi ${ }^{2,1}$ \\ ${ }^{1}$ Euro-Mediterranean Center on Climate Change (CMCC), Lecce, Italy \\ ${ }^{2}$ Department of Physics and Astronomy, Alma Mater Studiorum University of Bologna, Bologna, Italy \\ Correspondence to: Eric Jansen (eric.jansen@cmcc.it)
}

Received: 17 March 2016 - Published in Nat. Hazards Earth Syst. Sci. Discuss.: 30 March 2016

Accepted: 5 July 2016 - Published: 27 July 2016

\begin{abstract}
On 7 March 2014 (UTC), Malaysia Airlines flight 370 vanished without a trace. The aircraft is believed to have crashed in the southern Indian Ocean, but despite extensive search operations the location of the wreckage is still unknown. The first tangible evidence of the accident was discovered almost 17 months after the disappearance. On 29 July 2015, a small piece of the right wing of the aircraft was found washed up on the island of Réunion, approximately $4000 \mathrm{~km}$ from the assumed crash site. Since then a number of other parts have been found in Mozambique, South Africa and on Rodrigues Island.

This paper presents a numerical simulation using highresolution oceanographic and meteorological data to predict the movement of floating debris from the accident. Multiple model realisations are used with different starting locations and wind drag parameters. The model realisations are combined into a superensemble, adjusting the model weights to best represent the discovered debris. The superensemble is then used to predict the distribution of marine debris at various moments in time. This approach can be easily generalised to other drift simulations where observations are available to constrain unknown input parameters.

The distribution at the time of the accident shows that the discovered debris most likely originated from the wide search area between 28 and $35^{\circ} \mathrm{S}$. This partially overlaps with the current underwater search area, but extends further towards the north. Results at later times show that the most probable locations to discover washed-up debris are along the African east coast, especially in the area around Madagascar. The debris remaining at sea in 2016 is spread out over a wide area and its distribution changes only slowly.
\end{abstract}

\section{Introduction}

Modern sea situational awareness technologies make use of both real-time information and advanced, long-term reconstructions of the ocean state. Among many other applications, the ocean reconstructions allow the study of transport and dispersal scenarios for objects and pollutants at sea. Such studies are key to preparing better emergency response management plans and performing post-crisis assessments. The use of numerical simulations for search-and-rescue modelling dates back to the early 1970s, when the US Coast Guard introduced its Computer-Assisted Search Planning system (Frost and Stone, 2001). Developments in meteorology, oceanography and computer technology have since led to large improvements in the performance and accuracy of the numerical models. However, the basic methodology of drift modelling has remained the same, using an ensemble of particles that respond linearly to the wind speed (Hackett et al., 2006; Breivik et al., 2012). The relationship between drift and wind speed has been the subject of several decades worth of field experiments, ultimately leading to a system of standardised coefficients or leeway factors (Allen and Plourde, 1999).

The use of superensembles, a weighted combination of multiple models, was first introduced in meteorology by $\mathrm{Kr}$ ishnamurti et al. (1999) as a method to create multi-model forecasts. Applications in oceanography and more specifically in surface drift modelling (Rixen and Ferreira-Coelho, 2007; Rixen et al., 2008) have so far focused on improving the forcing fields used as input to the drift model. This paper presents a different approach, applying the superensemble technique to multiple realisations of the drift model itself. Using long-term global ocean and weather reconstructions, the superensemble drift model is used to produce probability 
distributions for the surface drift of aircraft debris originating from an accident in the southern Indian Ocean.

Malaysia Airlines flight 370 (MH370) was a scheduled passenger flight from Kuala Lumpur, Malaysia, to Beijing, China. The Boeing 777-200 ER aircraft carrying 239 passengers and crew disappeared less than an hour after take-off. Air traffic control (ATC) lost voice and radar contact with the flight at 17:22 UTC on 7 March 2014, while the aircraft was over the Gulf of Thailand. Initially it was assumed that the flight had crashed, but analysis of military radar data showed that the plane had deviated from its planned flight path and returned towards Malaysia. The aircraft continued flying in a westward direction and eventually exited the radar coverage at 18:22 UTC (Safety Investigation Team for MH370, 2015).

While most on-board communication equipment was inoperable, minimal communication between the aircraft's satellite terminal and the satellite network continued until 00:19 UTC. Analysis of this communication (Ashton et al., 2015) concluded that the aircraft changed course towards the south and continued in this direction until fuel ran out. Based on this information, various search areas have been defined in the southern Indian Ocean in an effort to locate the wreckage (ATSB, 2015). These areas straddle the arc of constant distance to the satellite at the time of the final communication. The most important areas are shown in Fig. 1. The wide search area is the envelope of possible locations, but due to its size a full search of this area is not feasible. Motivated by the most probable flight path, underwater searches for the wreckage have concentrated in the southern part of this area. However, the main wreckage has not been located. The only parts of the aircraft that have been recovered so far are the following:

- a flaperon, part of the right wing of the aircraft, found on the beach of Réunion on 29 July 2015;

- a flap fairing from the right wing, found in Mozambique in December 2015, but only reported to the authorities in March 2016 after media coverage of other debris (ATSB, 2016a);

- part of an engine cowling, discovered in South Africa in March 2016, but later found to be photographed already on 23 December 2015 (ATSB, 2016b);

- part of the horizontal stabiliser, found on a sandbank off the coast of Mozambique on 27 February 2015 (ATSB, 2016a);

- a cabin interior panel, discovered on Rodrigues Island, Mauritius, on 30 March 2016 (ATSB, 2016b).

The following sections will present a numerical simulation that uses all available information about the crash site and the discoveries of washed-up debris to predict the distribution of floating debris from the accident. The paper is organised as follows: in Sect. 2 the drift modelling and the treatment of

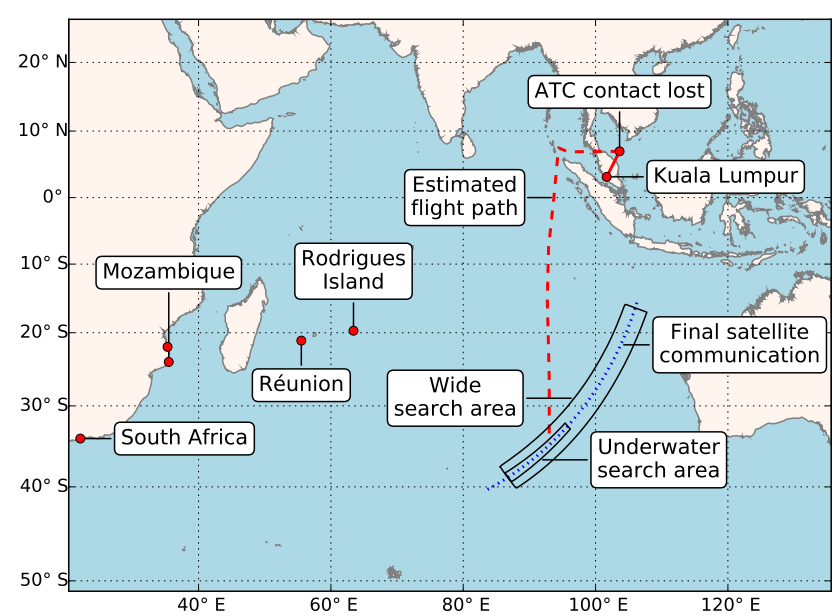

Figure 1. Estimated flight path of flight MH370 based on military radar and satellite data analysis. Also indicated are the search areas and the locations where washed-up debris was discovered.

unknown initial conditions is discussed; Sect. 3 shows the predictions of the model in terms of a time series of debris probability maps; and finally, Sect. 4 presents the conclusions of this study.

\section{Modelling}

\subsection{Drift trajectories}

Drift trajectories are modelled using an ensemble of particles drifting on the ocean surface. Due to differences in their initial positions and due to random motion these particles will slowly diverge over time. Each trajectory represents a possible path of the object being studied.

In this model the displacement $\mathrm{d} \boldsymbol{x}_{i}$ of particle $i$ in an infinitesimal time interval $\mathrm{d} t$ is given by

$\mathrm{d} \boldsymbol{x}_{i}=\left[\boldsymbol{c}\left(\boldsymbol{x}_{i}, t\right)+L \boldsymbol{w}\left(\boldsymbol{x}_{i}, t\right)\right] \mathrm{d} t+\boldsymbol{n}$.

Here $\boldsymbol{c}\left(\boldsymbol{x}_{i}, t\right)$ and $\boldsymbol{w}\left(\boldsymbol{x}_{i}, t\right)$ are the ocean current and wind forcing fields, interpolated at position $\boldsymbol{x}_{i}$ and time $t$. The constant $L$ is the wind drag coefficient, or downwind leeway (Allen and Plourde, 1999) of the particle. It accounts for both direct and indirect (e.g. Stokes drift) wind-induced motion. The term $\boldsymbol{n}$ parameterises the incoherent turbulent motion of the particle. Its components $n_{j}$ are random variables, distributed according to

$n_{j} \sim \mathcal{N}\left(0, \sqrt{2 K_{h} \Delta t}\right)$

$K_{h}=2 \mathrm{~m}^{2} \mathrm{~s}^{-1}$,

where $\mathcal{N}(\mu, \sigma)$ is the normal distribution and $\Delta t$ the length of an integration time step. The constant $K_{h}$ is the turbulent diffusion coefficient. The value of this coefficient is taken from De Dominicis et al. (2013). 
The particles in the model are advected by integrating Eq. (1) numerically using the fourth-order Runge-Kutta method.

\subsection{Superensemble}

To account for the fact that the location of the wreckage and the wind drag coefficient are not accurately known, the results presented here use multiple realisations of the model, with varying initial conditions and parameters. The different realisations are then combined into a superensemble.

The superensemble probability $P(x, t)$ of finding aircraft debris in a location $\boldsymbol{x}$ at time $t$ is expressed as a sum over all model realisations $k$ and their individual probabilities $P_{k}$ as

$P(\boldsymbol{x}, t)=\sum_{k} \alpha_{k} P_{k}(\boldsymbol{x}, t)$,

where $\alpha_{k}$ is the model weight coefficient. The probability predicted by the $k$ th model realisation can be defined as

$P_{k}(\boldsymbol{x}, t)=\frac{1}{N_{k}} \sum_{i=1}^{N_{k}} \delta\left(\boldsymbol{x}-\boldsymbol{x}_{k, i}(t)\right)$,

where $N_{k}$ is the total number of particles and $\delta$ a function that equals 1 below a certain distance and 0 otherwise. For all debris discoveries this distance is set to $100 \mathrm{~km}$.

\subsubsection{Model coefficients}

In order to obtain meaningful results from the superensemble, the coefficients $\alpha_{k}$ are chosen to represent the likelihood of a particular model realisation $M_{k}$, given the observed debris $D$. Using Bayesian inference $\alpha_{k}$ can be expressed as

$\alpha_{k}=\mathcal{P}\left(M_{k} \mid D\right) \propto \mathcal{P}\left(D \mid M_{k}\right) \times \mathcal{P}\left(M_{k}\right)$.

Here $\mathcal{P}\left(\mathrm{D} \mid M_{k}\right)$ can be obtained as a product of probabilities of the individual debris discoveries $l$ using Eq. (5):

$\mathcal{P}\left(D \mid M_{k}\right)=\prod_{l} P_{k}\left(\boldsymbol{x}_{l}, t_{l}\right)$.

The prior model likelihood $\mathcal{P}\left(M_{k}\right)$ is chosen inversely proportional to the model variance:

$\mathcal{P}\left(M_{k}\right)=\frac{1 / \sigma_{x, k}^{2}}{\sum_{n} 1 / \sigma_{x, n}^{2}}$.

The variance is evaluated at time $t=90$ days to capture the variability of the different model realisations without particles stranding on the coastline.

\subsubsection{Initial conditions and parameters}

The superensemble is initialised with 30 model realisations, each containing 5000 particles. The realisations contain all possible combinations of six different wind drag coefficients and five starting locations. The wind drag coefficients range from 0 to $2.5 \%$ in increments of $0.5 \%$, spanning roughly the range from no wind drag at all to a drag similar to that of a small boat (Allen and Plourde, 1999). The starting locations are obtained by dividing the wide search area (see Fig. 1) in five sections of equal size, along the arc of the final satellite communication.

\subsection{Forcing field data}

The oceanographic and meteorological data for the simulation are provided by the European Copernicus Marine Environment Monitoring Service ${ }^{1}$. The ocean currents at the surface level are extracted from the global ocean analysis ${ }^{2}$ data, which provide a three-dimensional ocean reconstruction with global coverage at a spatial resolution of $1 / 12^{\circ}$. The temporal resolution for the period of the simulation is 2-hourly, except for the period of 8-18 March 2014, where the daily average is used. The wind forcing uses the global near-real-time ocean wind observations ${ }^{3}$, which consist of satellite scatterometer observations combined with the operational wind analysis from the European Centre for Medium-Range Weather Forecasts (ECMWF). The wind fields are measured as a $6 \mathrm{~h}$ time average with a spatial resolution of $1 / 4^{\circ}$. The wind speed is defined at a reference height of $10 \mathrm{~m}$.

\section{Results}

\subsection{First debris discovery}

Figure 2 shows the distributions for floating MH370 debris in the southern Indian Ocean, using only the first observation in July 2015 to calculate the weights. The amount of debris predicted in a certain location is expressed as the number of simulated drifting particles in an area of $1^{\circ} \times 1^{\circ}$. The number of particles is normalised to that used in a single model realisation, i.e. $\sum_{k} \alpha_{k}=1$. Areas containing fewer than two particles per square degree are not shown.

The distribution at the time of the discovery in Réunion is shown in Fig. 2a. In addition to Réunion and the neighbouring islands, it shows a high probability of debris washed up in the northeast of Madagascar and on the African mainland in Tanzania and Mozambique. It also shows the probability of debris drifting east from the search area, ending up on the coast of southwest Australia.

In February 2016 (see Fig. 2b) the debris had spread further south along the African coast, reaching as far as South Africa. This distribution shows good agreement with the debris that was discovered roughly around this time on Rodrigues Island and in Mozambique. The engine cowling frag-

\footnotetext{
${ }^{1}$ http://marine.copernicus.eu.

${ }^{2}$ Dataset global-analysis-forecast-phys-001-002.

${ }^{3}$ Dataset wind-glo-wind-14-nrt-observations-012-004.
} 

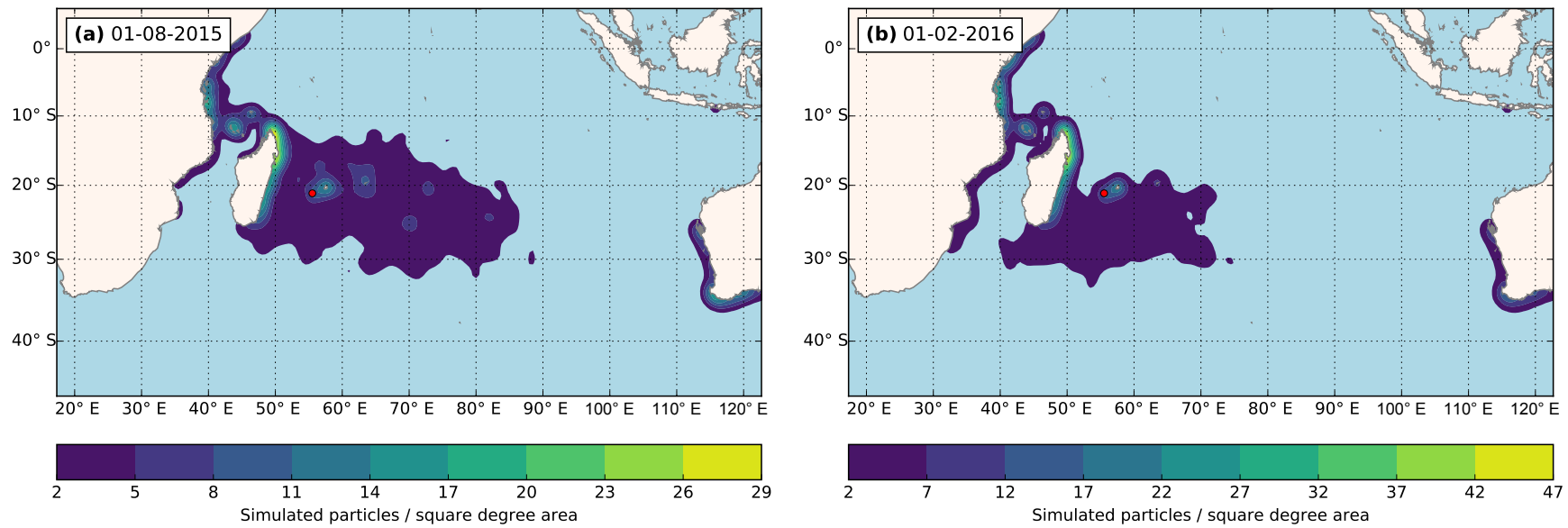

Figure 2. Floating aircraft debris probability density in (a) August 2015 and (b) February 2016, based only on the first observed debris at the end of July 2015. The location of this observation is indicated by the circular red marker.
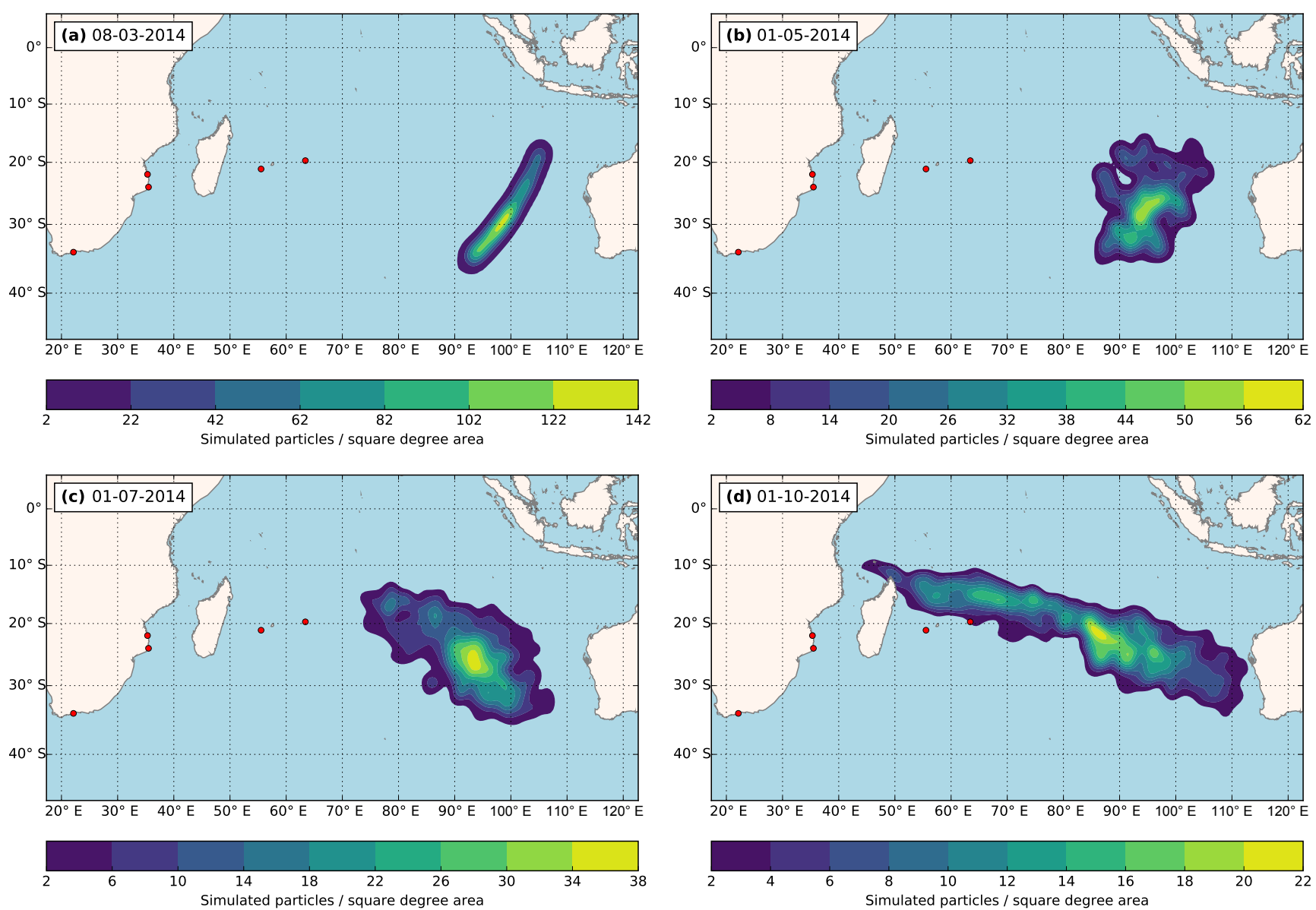

Figure 3. Floating aircraft debris probability density between March and October 2014. The superensemble weights are calculated based on all five debris observations. The locations of the observations are indicated by the circular red markers. 

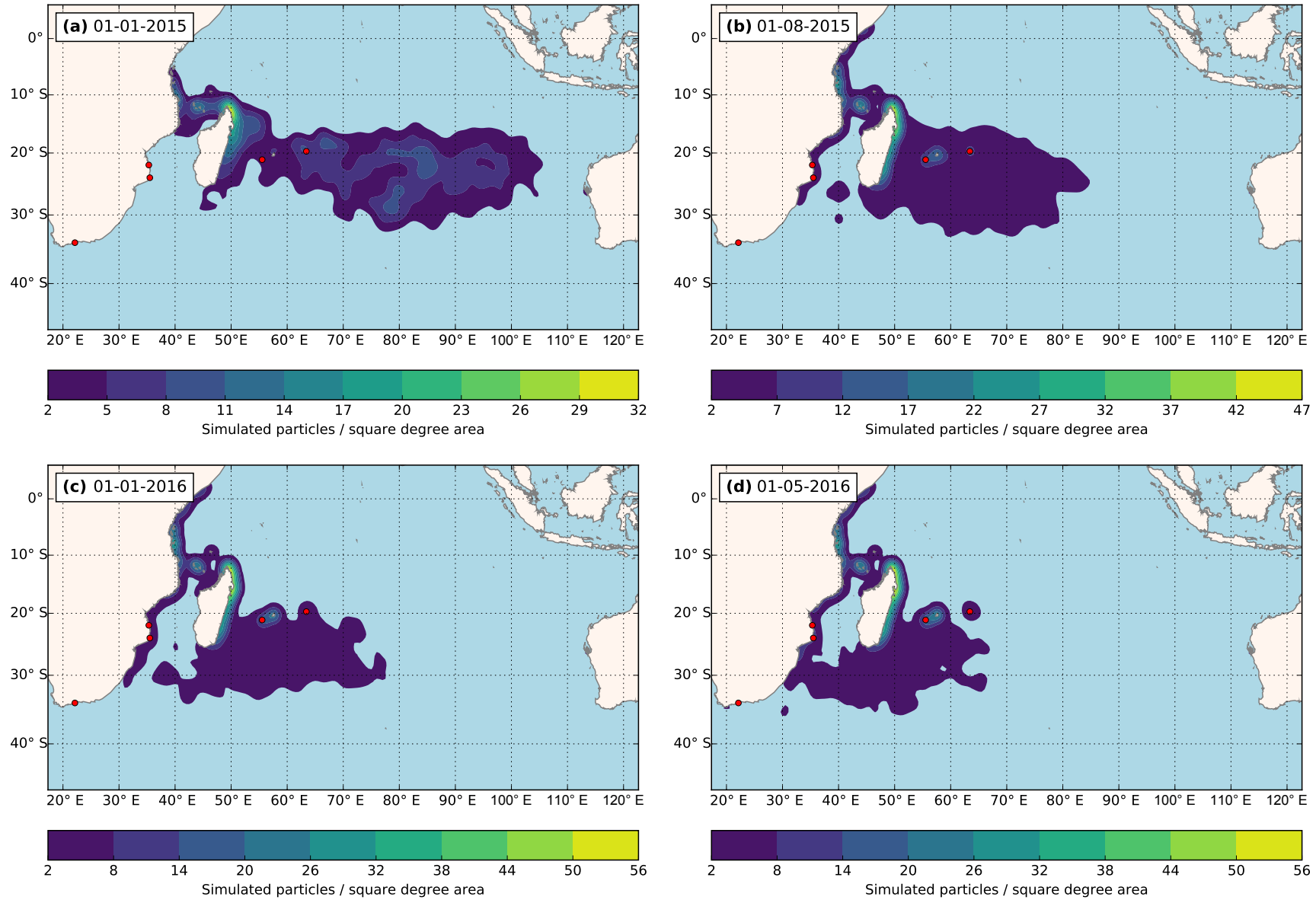

Figure 4. Floating aircraft debris probability density between January 2015 and May 2016. The superensemble weights are calculated based on all five debris observations. The locations of the observations are indicated by the circular red markers.

ment was discovered further south in South Africa, in a region where the simulation predicts fewer than two particles per square degree area.

\subsection{Including further discoveries}

The new debris discoveries can be included into the simulation by recalculating the model weights $\alpha_{k}$, resulting in the most likely distribution of debris that is compatible with all five observations. The time evolution of this distribution is shown in Figs. 3-4.

One density distribution that is of particular interest is the distribution at the time of the accident, shown in Fig. 3a. It shows that the most likely origin for the discovered debris ( $>100$ simulated particles per square degree) coincides with the northern half of the underwater search area, but extends further north until approximately $28^{\circ} \mathrm{S}$. Debris originating from the southern part of the underwater search area, below $35^{\circ} \mathrm{S}$, is found to be poorly compatible with the observed debris in Mozambique and South Africa.
Figure $3 b-d$ clearly show the anticlockwise pattern of the Indian Ocean gyre, with the West Australian Current moving north along the Australian coast and the westward South Equatorial Current around $20^{\circ} \mathrm{S}$. Figure $3 \mathrm{~d}$ shows that the first debris may have arrived on Rodrigues Island and the northern part of Madagascar as early as October 2014.

In January 2015 (Fig. 4a) the first debris may have reached Réunion. At roughly the same time debris is also predicted to have reached the African mainland, mostly on the coast of Tanzania and Mozambique. From this point on the debris entered the Agulhas Current that flows south along the African coast, possibly carrying debris as far as South Africa at the beginning of 2016 (Fig. 4c). The debris that remains at sea in 2016 (Fig. 4d) is spread out over a large area, and its distribution changes only slowly.

While debris may wash up on shore in various locations, it is important to note that there are many other factors that determine whether the debris will be found, recognised and reported to the proper authorities. In sparsely populated areas it may take a long time before debris is discovered, while in developing countries a discovered piece of debris may be 
discarded because people are not aware of the missing aircraft. It is therefore not unlikely that debris has washed up in other locations but has remained unreported.

\section{Conclusions}

The availability of high-resolution oceanographic and meteorological data is key to adequately responding to accidents and emergencies at sea. The case of MH370 especially underlines the importance of data that provide global coverage. With the operational range of modern airliners in excess of $10000 \mathrm{~km}$, accidents may happen in remote areas that are deprived of most means of communication. In such cases the meteo-oceanographic data might be the only source of information on the circumstances of the accident and the conditions afterwards.

The results presented in this paper show that the aircraft debris discovered so far in Réunion, Mozambique, South Africa and Rodrigues Island most likely originated from the wide search area between 28 and $35^{\circ} \mathrm{S}$. This is in agreement with the estimated flight path of Ashton et al. (2015) and partially overlaps with the underwater search area between 32 and $35^{\circ} \mathrm{S}$. However, this area extends further north than the current underwater search area, suggesting that if the current search proves unsuccessful, a northward extension of the search area should be considered.

Based on the particle distribution, the most probable locations to discover further washed-up debris from flight MH370 are Tanzania, Mozambique, Madagascar and surrounding islands such as Réunion, Mauritius and the Comoros. The time evolution shows that from 2016 onwards the floating debris that remains at sea is spread out, and its distribution changes only slowly. The probability of new debris washing up in the future is therefore quite low.

The results obtained for MH370 show that the superensemble method described in this paper is well-suited for drift simulations where additional observations are available to refine the result. By adjusting the weight of the superensemble members it is straightforward to incorporate information such as the discovered debris into the simulation. Moreover, as this weighting procedure is computationally inexpensive, the method could also be used to provide real-time operational forecasts during an emergency at sea.

Acknowledgements. This work was performed in the framework of the TESSA Project (Sviluppo di TEcnologie per la Situational Sea Awareness) supported by PON (Ricerca \& Competitività 20072013), cofunded by EU (European Regional Development Fund), MIUR (Ministero Italiano dell'Università e della Ricerca) and MSE (Ministero dello Sviluppo Economico). This study has been conducted using EU Copernicus Marine Service Information.
Edited by: R. Archetti

Reviewed by: two anonymous referees

\section{References}

Allen, A. A. and Plourde, J. V.: Review of leeway: field experiments and implementation, Tech. Rep. CG-D-08-99, US Coast Guard Research and Development Center, Groton, CT, USA, 1999.

Ashton, C., Shuster Bruce, A., Colledge, G., and Dickinson, M.: The search for MH370, J. Navigat., 68, 1-22, doi:10.1017/S037346331400068X, 2015.

ATSB: MH370 - Definition of underwater search areas, Tech. Rep. AE-2014-054, Australian Transportation Safety Bureau, Canberra, ACT, Australia, 2015.

ATSB: Debris examination - update No. 1, Tech. Rep. AE-2014054, Australian Transportation Safety Bureau, Canberra, ACT, Australia, 2016a.

ATSB: Debris examination - update No. 2, Tech. Rep. AE-2014054, Australian Transportation Safety Bureau, Canberra, ACT, Australia, 2016b.

Breivik, Ø., Allen, A. A., Maisondieu, C., and Olagnon, M.: Advances in search and rescue at sea, Ocean Dynam., 63, 83-88, doi:10.1007/s10236-012-0581-1, 2012.

De Dominicis, M., Pinardi, N., Zodiatis, G., and Lardner, R.: MEDSLIK-II, a Lagrangian marine surface oil spill model for short-term forecasting - Part 1: Theory, Geosci. Model Dev., 6, 1851-1869, doi:10.5194/gmd-6-1851-2013, 2013.

Frost, J. and Stone, L.: Review of search theory: advances and applications to search and rescue decision support, Tech. Rep. CGD-15-01, US Coast Guard Research and Development Center, Canberra, ACT, Australia, 2001.

Hackett, B., Breivik, Ø., and Wettre, C.: Forecasting the drift of objects and substances in the ocean, in: Ocean weather forecasting, vol. 5, chap. 23, edited by: Chassignet, E. P. and Verron, J., Springer Netherlands, 507-523, doi:10.1007/1-4020-4028-8_23, 2006.

Krishnamurti, T. N., Kishtawal, C. M., LaRow, T. E., Bachiochi, D. R., Zhang, Z., Williford, C. E., Gadgil, S., and Surendran, S.: Improved weather and seasonal climate forecasts from multimodel superensemble, Science, 285, 1548-1550, doi:10.1126/science.285.5433.1548, 1999.

Rixen, M. and Ferreira-Coelho, E.: Operational surface drift prediction using linear and non-linear hyper-ensemble statistics on atmospheric and ocean models, J. Mar. Syst., 65, 105-121, doi:10.1016/j.jmarsys.2004.12.005, 2007.

Rixen, M., Ferreira-Coelho, E., and Signell, R.: Surface drift prediction in the Adriatic Sea using hyper-ensemble statistics on atmospheric, ocean and wave models: uncertainties and probability distribution areas, J. Mar. Syst., 69, 86-98, doi:10.1016/j.jmarsys.2007.02.015, 2008.

Safety Investigation Team for MH370: factual information - safety investigation for MH370, Tech. Rep. MH370/01/15, Ministry of Transport, Malaysia, 2015. 